\title{
First-principles investigation of graphene fluoride and graphane
}

\author{
O. Leenaerts, ${ }^{1, *}$ H. Peelaers, ${ }^{1, \dagger}$ A. D. Hernández-Nieves, ${ }^{1,2, \ddagger}$ B. Partoens, ${ }^{1, \S}$ and F. M. Peeters ${ }^{1, \|}$ \\ ${ }^{1}$ Departement Fysica, Universiteit Antwerpen, Groenenborgerlaan 171, B-2020 Antwerpen, Belgium \\ ${ }^{2}$ Centro Atomico Bariloche, 8400 San Carlos de Bariloche, Rio Negro, Argentina
}

(Received 20 September 2010; revised manuscript received 18 October 2010; published 18 November 2010)

\begin{abstract}
Different stoichiometric configurations of graphane and graphene fluoride are investigated within densityfunctional theory. Their structural and electronic properties are compared, and we indicate the similarities and differences among the various configurations. Large differences between graphane and graphene fluoride are found that are caused by the presence of charges on the fluorine atoms. A configuration that is more stable than the boat configuration is predicted for graphene fluoride. We also perform GW calculations for the electronic band gap of both graphene derivatives. These band gaps and also the calculated Young's moduli are at variance with available experimental data. This might indicate that the experimental samples contain a large number of defects or are only partially covered with $\mathrm{H}$ or F.
\end{abstract}

DOI: 10.1103/PhysRevB.82.195436

PACS number(s): 61.48.Gh, 68.43.-h, 73.22.Pr, 81.05.ue

\section{INTRODUCTION}

Two-dimensional crystals have been given a large amount of attention since the isolation of one-atom-thick materials by Novoselov et al. in 2004. ${ }^{1,2}$ Graphene, a single layer of graphite, has attracted by far the most attention because of the high crystal quality of the graphene samples and its fascinating electronic properties. ${ }^{3}$ These properties make it a promising candidate to use as a basic material for future electronics applications. ${ }^{4}$ However, the use of graphene for applications in electronics suffers from a major drawback: graphene is, in its pristine state, a zero-band-gap semiconductor and this gapless state appears to be rather robust. Several ways have been explored to induce a finite band gap in graphene. It was found experimentally that a band gap can be opened by confining the electrons in nanoribbons ${ }^{5}$ or by applying a potential difference over a graphene bilayer. 6,7

The chemical modification of graphene is another promising way to create a band gap. ${ }^{8-11}$ When radicals such as oxygen, hydrogen, or fluorine atoms are adsorbed on the graphene surface they form covalent bonds with the carbon atoms. These carbon atoms change their hybridization from $s p^{2}$ to $s p^{3}$, which leads to the opening of a band gap (similar as in diamond). The adsorbed radicals can attach to the graphene layer in a random way, as is the case in graphene oxide, ${ }^{12,13}$ or they can form ordered patterns. In the last case, new graphene-based two-dimensional (2D) crystals are formed with properties that can vary greatly from their parent material. This has been found to be the case for hydrogen and fluorine adsorbates. The new 2D crystals that are expected to form in those cases ${ }^{14}$ have been named graphane $^{8,15}$ and graphene fluoride (or fluorographene), ${ }^{11}$ respectively.

Following this route, multilayer graphene fluoride was recently synthesized, $, 9,10$ and its structural and electronic properties were studied. A strongly insulating behavior was found with a room-temperature resistance larger than $10 \mathrm{G} \Omega$, which is consistent with the existence of a large band gap in this new material. ${ }^{9,10}$ Only a partial fluorine coverage of the graphene multilayer samples was achieved in these experiments. The F/C ratio was estimated to be 0.7 in Ref. 9 and
0.24 in Ref. 10, according to weight gain measurements.

Another important step forward in creating fully covered two-dimensional graphene fluoride samples was recently achieved in Ref. 11. The obtained single-layer graphene fluoride exhibits a strong insulating behavior with a roomtemperature resistance larger than $1 \mathrm{~T} \Omega$, a strong temperature stability up to $400{ }^{\circ} \mathrm{C}$, and almost a complete disappearance of the graphene Raman peaks associated with regions that are not fully fluorinated. ${ }^{11}$ The graphene Raman peaks do not disappear completely, however, which could be an indication of the presence of defects in the sample, such as a small portion of carbon atoms not bonded to fluorine atoms. It was also found experimentally that fluorographene has a Young's modulus of $\approx 100 \mathrm{~N} / \mathrm{m}$ and the optical measurements suggest a band gap of $\approx 3 \mathrm{eV}$. In Ref. 16 it was demonstrated that single-side adsorption is also possible and that it probably results in a crystalline $\mathrm{C}_{4} \mathrm{~F}$ structure with a large band gap.

On the theoretical side, first-principles studies on graphene monofluoride started in 1993, motivated by available experiments on graphite monofluoride. ${ }^{17}$ Using densityfunctional theory (DFT) calculations, it was shown in Ref. 18 that the chair configuration of graphene fluoride is energetically more favorable than the boat configuration by 0.145 $\mathrm{eV}$ per $\mathrm{CF}$ unit $(0.073 \mathrm{eV} /$ atom $)$ while a transition barrier on the order of $2.72 \mathrm{eV}$ was found between both structures. Due to the small difference in formation energy and the large energy barrier between both configurations, it was argued that the kinematics of the intercalation could selectively determine the configuration, or that there could also be a mixing of both configurations in the available experiments. By using the local-density approximation for the exchangecorrelation functional a direct band gap of $3.5 \mathrm{eV}$ was calculated for the chair configuration in Ref. 18. However, it is well known that DFT generally underestimates the band gap. Recent calculations used the more accurate GW approximation and found a much larger band gap of $7.4 \mathrm{eV}$ for the chair configuration of graphene monofluoride (Ref. 19). This theoretical value is twice as large as the one obtained experimentally for graphene fluoride in Ref. 11 , which is $\approx 3 \mathrm{eV}$. The experimental value for the Young's modulus as found in Ref. $11(\approx 100 \mathrm{~N} / \mathrm{m})$ is also half the value obtained recently 
from first-principles calculations in Ref. $20(\approx 228 \mathrm{~N} / \mathrm{m})$ for the chair configuration of graphene fluoride. It is worth noting that the experimental ${ }^{21}$ and theoretical ${ }^{22}$ values of the Young's modulus of graphene only differ in a small percentage.

Possible reasons for the disagreement between the experimental values and the $a b$ initio results for the Young's modulus and the band gap of graphene fluorine could be: (i) the presence of a different configuration or a mixture of them in the experimental samples or (ii) the presence of defects, which could decrease the size of both the Young's modulus and the band gap from the expected theoretical values.

In this paper, we investigate various possible crystal configurations for both graphene-based two-dimensional crystals, graphene fluoride and graphane, and we examine their structural, electronic, and mechanical properties. In the case of graphene fluorine, we found a distinct configuration that has a lower energy than the boat configuration. This configuration, which we call the zigzag configuration, is energetically less favorable than the chair configuration by only $0.073 \mathrm{eV}$ per $\mathrm{CF}$ unit (0.036 eV/atom). We calculated the Young's modulus and the band gap (both with generalized gradient approximation (GGA) and in the GW approximation) for the different configurations. The disagreements between experimental and $a b$ initio calculations for graphene fluoride persist independently of the considered configuration. These results imply that the available experimental samples probably contain a large number of defects, such as a portion of carbon atoms not bonded to fluorine atoms, that decrease the value of both the Young's modulus and the band gap from the expected theoretical values.

The paper is organized as follows: first we describe the computational details of our first-principles calculations. Then we investigate the stability and structural properties of the different configurations of both graphene derivatives. To conclude, the elastic and electronic properties of the different structures are discussed.

\section{COMPUTATIONAL DETAILS}

We examine different graphane and graphene fluoride configurations with the use of $a b$ initio calculations performed within the DFT formalism. The GGA of Perdew, Burke, and Ernzerhof ${ }^{23}$ is used for the exchange-correlation functional and a plane-wave basis set with a cutoff energy of 40 hartree is applied. The sampling of the Brillouin zone is done with the equivalent of a $24 \times 24 \times 1$ Monkhorst-Pack $k$-point grid $^{24}$ for a graphene unit cell and we use pseudopotentials of the Troullier-Martins type. ${ }^{25}$ Since periodic boundary conditions are applied in all three dimensions a vacuum layer of $20 \mathrm{bohr}$ is included to minimize the (artificial) interaction between adjacent layers. All the calculations were performed with the ABINIT code. ${ }^{26}$

The reported quasiparticle corrections for the band gap are obtained using the YAMBO code. ${ }^{27}$ Here the first-order quasiparticle corrections are obtained using Hedin's GW approximation $^{28}$ for the electron self-energy. Because we are treating two-dimensional systems, the spurious Coulomb interaction between a layer and its images should be avoided,

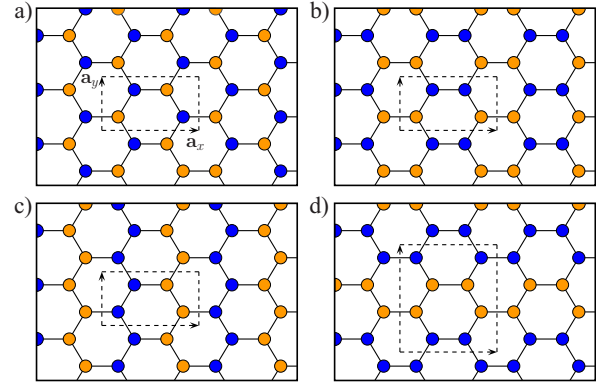

FIG. 1. (Color online) Four different configurations of hydrogen/fluorine-graphene: (a) chair, (b) boat, (c) zigzag, and (d) armchair configurations. The different colors (shades) represent adsorbates ( $\mathrm{H}$ or $\mathrm{F}$ ) above and below the graphene plane. The supercell used to calculate the elastic constants is indicated by the dashed box.

as this causes serious convergence problems. Therefore we use a truncation of this interaction in a box layout, following the method of Rozzi et $a .^{29}$ The remaining singularity is treated using a random integration method in the region near the gamma point. ${ }^{27}$ Nevertheless, a larger separation between the layers is necessary, so a value of $60 \mathrm{bohr}$ is used for these calculations.

\section{RESULTS}

We studied four different stoichiometric configurations for both graphane and graphene fluoride in which every carbon atom is covalently bonded to an adsorbate in an equivalent way, i.e., every carbon/adsorbate pair has the same environment. These configurations are schematically depicted in Fig. 1 and we will refer to them as the "chair," "boat," "zigzag," and "armchair" configuration. The chair and boat configurations have been well investigated before but the zigzag and armchair configurations are rarely examined for graphane ${ }^{14}$ and we are not aware of any studies for fluorographene. The names of these last two configurations have been chosen for obvious reasons [see Figs. 1(c) and 1(d)]. After relaxation, the different configurations appear greatly distorted when compared with the schematic pictures of Fig. 1, so these figures should only be regarded as topologically correct (see Fig. 2).

\section{A. Stability analysis}

To examine the stability of the different configurations, we make use of the formation energy of the structures and the binding energy between the graphene layer and the adsorbates. We define the formation energy, $E_{\mathrm{f}}$, as the energy per atom of the hydrogenated or fluorinated graphene with respect to intrinsic graphene and the corresponding diatomic molecules $\mathrm{H}_{2}$ and $\mathrm{F}_{2}$. The binding energy, $E_{\mathrm{b}}$, is defined with respect to graphene and the atomic energies of the adsorbates and is calculated per $\mathrm{CH}$ or $\mathrm{CF}$ pair. The results are summarized in Table I.

As has been reported before, the chair configuration is the most stable one for both graphane ${ }^{15}$ and graphene fluoride. ${ }^{18}$ The zigzag configuration is found to be more stable than the 

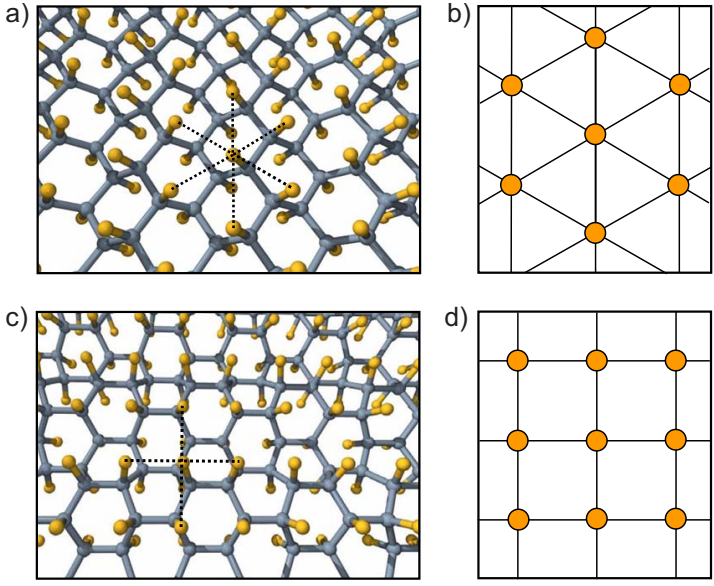

FIG. 2. (Color online) Fluorographene in the (a) zigzag and (c) armchair configurations. The nearest-neighbor bonds of one $\mathrm{F}$ atom are indicated with dotted lines to show the symmetry of the superlattices [as shown in (b) and (d)]. (b) The hexagonal superlattice which is formed in case of chair and zigzag configurations. (d) The cubic superlattice which is formed in case of boat and armchair configurations.

boat and armchair configurations and its formation energy is only slightly higher than that of the chair configuration: for both graphene derivatives the difference in formation energy, $\Delta E_{\mathrm{f}}$, between chair and zigzag is on the order of the thermal energy at room temperature $(26 \mathrm{meV})$. The energy differences between the various configurations are more pronounced for graphene fluorine than for graphane but they are of the same order of magnitude.

When we compare graphane and fluorographene, the binding energy of hydrogen and fluorine appears to be rather similar $(2.5 \mathrm{eV}$ compared to $2.9 \mathrm{eV})$ but there is a huge difference in the formation energy $(0.1 \mathrm{eV}$ compared to 0.9 $\mathrm{eV}$ ). This is a consequence of the large difference in the dissociation energy between hydrogen and fluorine molecules. The formation energy as defined above can be regarded as a measure of the stability against molecular desorption from the graphene surface. Therefore graphene fluoride is expected to be much more stable than graphane as has indeed been observed experimentally. ${ }^{8,11}$

TABLE I. The formation energy $E_{\mathrm{f}}$, the binding energy $E_{\mathrm{b}}$, and the relative binding energy $\Delta E_{\mathrm{f}}$ (with respect to the most stable configuration) for different hydrogenated and fluorinated graphene configurations. The energies are given in $\mathrm{eV}$.

\begin{tabular}{lcccc}
\hline \hline & Chair & Boat & Zigzag & Armchair \\
\hline \multicolumn{5}{c}{ Graphane } \\
$E_{\mathrm{b}}$ & -2.481 & -2.378 & -2.428 & -2.353 \\
$E_{\mathrm{f}}$ & -0.097 & -0.046 & -0.071 & -0.033 \\
$\Delta E_{\mathrm{f}}$ & 0.000 & 0.051 & 0.027 & 0.064 \\
& \multicolumn{5}{c}{ Fluorographene } \\
& -2.864 & -2.715 & -2.791 & -2.673 \\
$E_{\mathrm{b}}$ & -0.808 & -0.733 & -0.772 & -0.712 \\
$E_{\mathrm{f}}$ & 0.000 & 0.075 & 0.036 & 0.095 \\
$\Delta E_{\mathrm{f}}$ &
\end{tabular}

TABLE II. Structure parameters for the different hydrogenated and fluorinated graphene derivatives. Distances are given in $\AA$ and angles in degrees. The distance between neighboring $\mathrm{C}$ atoms, $d_{\mathrm{CC}}$, and the angles, $\theta_{\mathrm{CCX}}$, are averaged over the supercell.

\begin{tabular}{lcccc}
\hline \hline & Chair & Boat & Zigzag & Armchair \\
\hline \multicolumn{5}{c}{ Graphane } \\
$a_{x} / \sqrt{3}$ & 2.539 & 2.480 & 2.203 & 2.483 \\
$a_{y} / n_{y}$ & 2.539 & 2.520 & 2.540 & 2.270 \\
$d_{\mathrm{CH}}$ & 1.104 & 1.099 & 1.099 & 1.096 \\
$\bar{d}_{\mathrm{CC}}$ & 1.536 & 1.543 & 1.539 & 1.546 \\
$\bar{\theta}_{\mathrm{CCH}}$ & 107.4 & 107.0 & 106.8 & 106.7 \\
$\bar{\theta}_{\mathrm{CCC}}$ & 111.5 & 111.8 & 112.0 & 112.1
\end{tabular}

\begin{tabular}{lcccc}
$a_{x} / \sqrt{3}$ & 2.600 & 2.657 & 2.415 & 2.662 \\
$a_{y} / n_{y}$ & 2.600 & 2.574 & 2.625 & 2.443 \\
$d_{\mathrm{CF}}$ & 1.371 & 1.365 & 1.371 & 1.365 \\
$\bar{d}_{\mathrm{CC}}$ & 1.579 & 1.600 & 1.585 & 1.605 \\
$\bar{\theta}_{\mathrm{CCF}}$ & 108.1 & 106.0 & 104.6 & 104.2 \\
$\bar{\theta}_{\mathrm{CCC}}$ & 110.8 & 112.8 & 113.9 & 114.2 \\
\hline \hline
\end{tabular}

\section{B. Structural properties}

Besides the large difference in formation energy there are also pronounced structural differences between both graphene derivatives. The structural parameters for the different configurations of graphane and fluorographene are shown in Table II. Note that all the structures are described in an orthogonal supercell, as illustrated in Fig. 1, for ease of comparison. The results for the chair configuration agree well with previous theoretical calculations for graphane $e^{15,31,32}$ and graphene fluoride. ${ }^{30}$

It is also useful to compare the interatomic distances and bond angles with those of graphene and diamond. Therefore we calculated these using the same formalism as described above (Sec. II). The C-C bond has a length of $1.42 \AA$ for graphene compared to $1.54 \AA$ for diamond, and the bond angles are $120^{\circ}$ and $109.5^{\circ}$, respectively. Notice that both graphane and fluorographene resemble much closer the diamond structure than graphene, which is not surprising since the hybridization of the carbon atoms in these structures is the same as in diamond, i.e., $s p^{3}$. The $\mathrm{C}-\mathrm{C}$ bond length for the graphane configurations is similar to the one in diamond but $\bar{d}_{\mathrm{CC}}$ in fluorographene is about $0.05 \AA$ larger. This can be explained from a chemical point of view as due to a depopulation of the bonding orbitals between the carbon atoms. The depopulation of these bonding orbitals results from an electron transfer from the carbon to the fluorine atoms due to the difference in electronegativity between $\mathrm{C}$ and $\mathrm{F}$. We used a Hirshfeld-based method ${ }^{33-35}$ to calculate this charge transfer and found it to be $\Delta Q \approx 0.3 e$. The charge transfer in graphane is much smaller because of the similarity between the electronegativity of $\mathrm{C}$ and $\mathrm{H}$.

The fact that the fluorine atoms are negatively charged has an appreciable influence on the structure of graphene fluoride 
TABLE III. Elastic constants of the different hydrogenated and fluorinated graphene derivatives. The 2D Young's modulus, $E^{\prime}$, and Poisson's ratio, $\nu$, are given along the cartesian axes. $E^{\prime}$ is expressed in $\mathrm{N} \mathrm{m}^{-1}$.

\begin{tabular}{lcccc}
\hline \hline & Chair & Boat & Zigzag & Armchair \\
\hline \multicolumn{5}{c}{ Graphane } \\
$E_{x}^{\prime}$ & 243 & 230 & 117 & 247 \\
$E_{y}^{\prime}$ & 243 & 262 & 271 & 142 \\
$\nu_{x}$ & 0.07 & -0.01 & 0.05 & -0.05 \\
$\nu_{y}$ & 0.07 & -0.01 & 0.11 & -0.03 \\
& \multicolumn{5}{c}{ Fluorographene } \\
$E_{x}^{\prime}$ & 226 & 238 & 240 & 215 \\
$E_{y}^{\prime}$ & 226 & 240 & 222 & 253 \\
$\nu_{x}$ & 0.10 & 0.00 & 0.09 & 0.02 \\
$\nu_{y}$ & 0.10 & 0.00 & 0.11 & 0.02 \\
\hline \hline
\end{tabular}

when compared to graphane. This can, e.g., be seen from the sizes of the different bond angles. The bond angles (and also the bond lengths) in the chair configuration can be regarded as the ideal angles (lengths) for these structures because they can fully relax. The other configurations will try to adopt these ideal bond angles and it can be seen from Table II that this is indeed the case for the graphane configurations. The fluorographene configurations, on the other hand, appear to be somewhat distorted because their bond angles are (relatively) far from ideal. This is probably caused by the repulsion between the different fluorine atoms as can be demonstrated when focusing only on the positions of the $\mathrm{F}$ atoms. The fluorine atoms appear to form hexagonal or cubic superlattices depending on the configuration [see Figs. 2(b) and 2(d)]. This is trivial in the case of the chair (and maybe the boat) configuration but not so for the others [see Figs. 2(a) and 2(c)]. These superlattices are not perfect (deviations of a few percent) but are much more pronounced than in the case of graphane. So it seems that, at the cost of deforming the bonding angles, $\mathrm{F}$ superlattices are formed to minimize the electrostatic repulsion between the charged $\mathrm{F}$ atoms.

\section{Elastic strain}

Graphene and its derivatives graphane and fluorographene can be isolated and made into free-hanging membranes. This makes it possible to measure the elastic constants of these materials from nanoindentation experiments using an atomic force microscope..$^{8,11,21}$ The experimental elastic constants can be compared to first-principles calculations which gives us information about the purity and structural crystallinity of the experimental samples. Therefore we calculated ${ }^{36}$ the (2D) Young's modulus, $E^{\prime}$, and the Poisson's ratio, $\nu$, of the different graphane and fluorographene configurations, which we list in Table III. The Young's modulus and the Poisson's ratio of graphene are found to be $E^{\prime}=336 \mathrm{~N} \mathrm{~m}^{-1}$ and $\nu$ $=0.17$, respectively, which corresponds well to the experimental value, $E_{\exp }^{\prime}=340 \pm 50 \mathrm{~N} \mathrm{~m}^{-1}$, and other theoretical results. ${ }^{20,37,38}$
The 2D Young's modulus of graphane and fluorographene is smaller than that of graphene. The $E^{\prime}$ of the chair and boat configurations of both graphene derivatives are about $2 / 3$ the value of graphene which makes them very strong materials. The Young's modulus for the zigzag and armchair configurations of graphane are highly anisotropic with values that are roughly halved along the direction that shows the largest crumpling [see Figs. 2(a) and 2(c)]. The situation is completely different for fluorographene where the Young's modulus is more isotropic. This difference is probably caused by the deformations in the fluorographene configurations due to the charged $\mathrm{F}$ atoms. The values that are found for the chair configurations agree well with recent calculations $\left(245 \mathrm{~N} \mathrm{~m}^{-1}\right.$ and $228 \mathrm{~N} \mathrm{~m}^{-1}$ for graphane and fluorographene, respectively, in Ref. 20). Nair et al. ${ }^{11}$ performed a nanoindentation experiment on fluorographene and measured a value of $100 \pm 30 \mathrm{~N} \mathrm{~m}^{-1}$ for $E_{\mathrm{FG}}^{\prime}$. This value is approximately half the theoretical value. Because the theoretical values are trustworthy, i.e., they agree with earlier theoretical calculations, and this kind of calculations are believed to be accurate (as in the case of graphene), this suggests that the experimental samples contain an appreciable amount of defects. This conclusion is also supported by recent theoretical calculations on defected graphane in which it was demonstrated that even a small amount of vacancies $(1.6 \%)$ decreases the Young's modulus with $\approx 12 \%$. ${ }^{37} \mathrm{~A}$ similar situation might be expected for fluorographene but further theoretical and experimental research is needed for a better understanding of this influence of vacancies on the Young's modulus of graphene fluoride.

The Poisson's ratio shows a similar behavior as the Young's modulus and also agrees well with an earlier theoretical prediction of $\nu=0.07$ for the chair configuration of graphane. ${ }^{38}$ The knowledge of $E^{\prime}$ and $\nu$ allows us to calculate all the other 2D elastic constants ${ }^{39}$ such as the bulk, $K^{\prime}=E^{\prime} / 2(1-\nu)$, and shear modulus, $G^{\prime}=E^{\prime} / 2(1+\nu)$. For the chair configurations, we find $K_{\mathrm{HG}}^{\prime}=131 \mathrm{~N} \mathrm{~m}^{-1}$ and $G_{\mathrm{HG}}^{\prime}$ $=114 \mathrm{~N} \mathrm{~m}^{-1}$ for graphane, and $K_{\mathrm{FG}}^{\prime}=126 \mathrm{~N} \mathrm{~m}^{-1}$ and $G_{\mathrm{FG}}^{\prime}$ $=103 \mathrm{~N} \mathrm{~m}^{-1}$ for graphene fluoride.

\section{Electronic properties}

Graphene is a zero-gap semiconductor but its derivatives, such as graphane and fluorographene, have large band gaps, similar to diamond. In Table IV, the band gaps of the configurations under study are given. We also performed GW calculations because GGA is known to underestimate the band gap. The values for the chair configurations are in accordance with earlier theoretical calculations for graphane ${ }^{19,38,40}$ and fluorographene. ${ }^{19,40}$ The GGA and GW results show different behavior for the variation in the band gap among the different configurations. Note that this indicates that it is not straightforward to deduce qualitative trends from GGA as is often done in the literature. But, overall, we may conclude that the band gap is more or less independent of the configuration and that its size is roughly twice as large for GW as compared to GGA.

The GGA results give a band gap of $3.2 \mathrm{eV}$ for the most stable fluorographene configuration which is in accordance 
TABLE IV. Electronic properties of the different configurations of hydrogenated and fluorinated graphene derivatives. The electronic band gap, $E_{\text {gap }}$, is given for GGA and GW calculations. The IE is also calculated. All the energies are given in $\mathrm{eV}$.

\begin{tabular}{lcccc}
\hline \hline & Chair & Boat & Zigzag & Armchair \\
\hline \multirow{5}{*}{ Graphane } \\
$E_{\text {gap }}(\mathrm{GGA})$ & 3.70 & 3.61 & 3.58 & 3.61 \\
$E_{\text {gap }}(\mathrm{GW})$ & 6.05 & 5.71 & 5.75 & 5.78 \\
IE (GGA) & 4.73 & 4.58 & 5.30 & 4.65 \\
& \multicolumn{5}{c}{ Fluorographene } \\
& 3.20 & 3.23 & 3.59 & 4.23 \\
$E_{\text {gap }}(\mathrm{GGA})$ & 7.42 & 7.32 & 7.28 & 7.98 \\
$E_{\text {gap }}(\mathrm{GW})$ & 7.69 & 7.64 & 7.85 & 8.27 \\
IE (GGA) & \multicolumn{5}{c}{} \\
\hline \hline
\end{tabular}

with the experimental result of $\sim 3 \mathrm{eV}$ as found in Ref. 11 . However, this value is much smaller than the (more accurate) GW band gap of $7.4 \mathrm{eV}$ so that the theoretical and experimental results differ by about a factor of 2 . This conflict might be resolved if the experimental value is ascribed to midgap states due to defects in the system, such as missing $\mathrm{H} / \mathrm{F}$ atoms (similar to what has been predicted for defected graphane $\left.^{41}\right)$.

The electronic band structure and the corresponding density of states of graphane and fluorographene in the chair configuration are shown in Fig. 3. Both band structures look similar but there are also some clear differences. In the case of fluorographene the parabolic band at the $\Gamma$ point, corresponding to quasifree-electron states, is at much higher energies, which indicates a larger ionization energy (IE) for fluorinated graphene. This IE is defined as the difference between the vacuum level and the valence-band maximum and an explicit calculation of this energy indicates a difference of about $3 \mathrm{eV}$ between graphane and fluorographene (see Table IV). This is a consequence of the negative charges on the fluorine atoms in fluorographene. We can also compare the IE values with the work function of graphene which is the same as its ionization potential (because graphene has no band gap) and has a value of $4.22 \mathrm{eV}$ from GGA (this is somewhat smaller that the experimental value ${ }^{42}$ of $4.57 \pm 0.05 \mathrm{eV})$. It can be seen from Table IV that the ionization energies of both graphene derivatives are higher than that of graphene (differences of $\approx 0.5 \mathrm{eV}$ and $3.5 \mathrm{eV}$, respectively), although the ionization energy of graphane is rather similar to graphene.

\section{SUMMARY AND CONCLUSIONS}

We investigated different configurations of the graphene derivatives fluorographene and graphane. The chair configu-
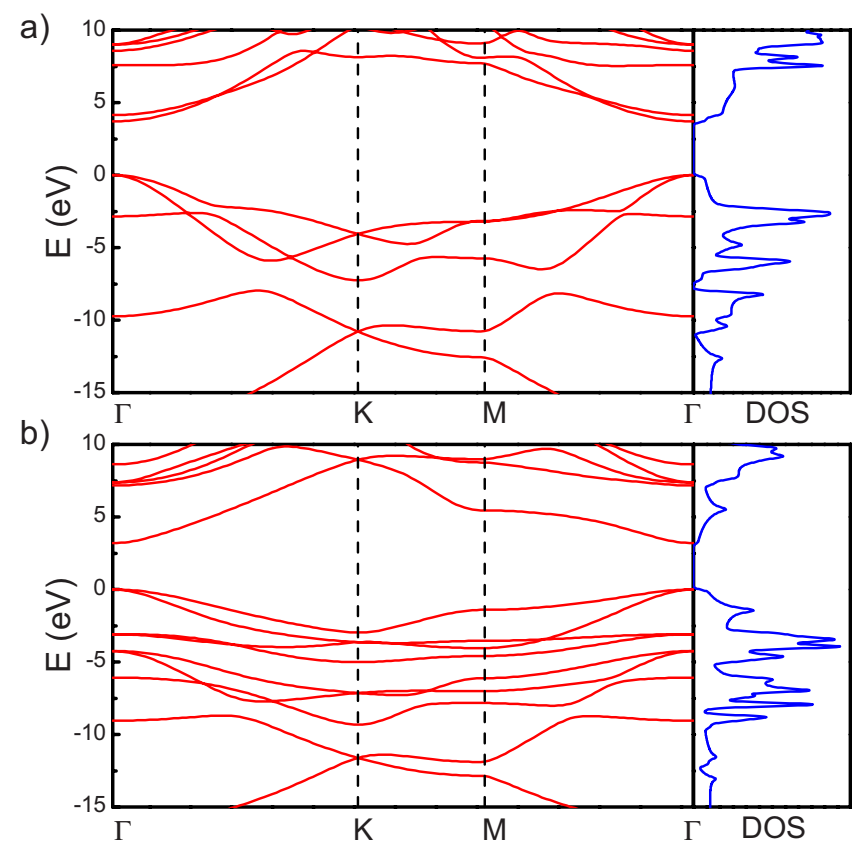

FIG. 3. (Color online) The electronic band structure and the corresponding density of states (GGA) for the chair configuration of (a) graphane and (b) fluorographene. The valence-band maximum has been used as the origin of the energy scale.

ration is the most stable one in both cases but the zigzag configuration has only a slightly higher formation energy and is more stable than the much more studied boat configuration. Fluorographene is found to be much more stable than graphane which is mainly due to a much higher desorption energy for $\mathrm{F}_{2}$ as compared to $\mathrm{H}_{2}$. We also demonstrated that there are structural and electronic differences that are caused by the charged state of the $\mathrm{F}$ atoms in fluorographene.

When our results are compared to available experimental data for fluorographene some discrepancies can be noticed: for all the configurations studied we find much larger band gaps in the electronic band structure and the calculated Young's modules is much larger. This might indicate that the experimental samples still contain appreciable amounts of defects. The nature of these defects requires further investigation but one can speculate that these defects consist of missing adsorbates, partial $\mathrm{H} / \mathrm{F}$ coverage, or mixed configurations.

\section{ACKNOWLEDGMENTS}

This work was supported by the Flemish Science Foundation (FWO-Vl), the NOI-BOF of the University of Antwerp, the Belgian Science Policy (IAP), and the collaborative project FWO-MINCyT (Grant No. FW/08/01). A.D.H. also acknowledges support from ANPCyT (Grant No. PICT 2008-2236). 
*ortwin.leenaerts@ua.ac.be

†hartwin.peelaers@ua.ac.be

*alexande@cab.cnea.gov.ar

§bart.partoens@ua.ac.be

"francois.peeters@ua.ac.be

${ }^{1}$ K. S. Novoselov, A. K. Geim, S. V. Morozov, D. Jiang, Y. Zhang, S. V. Dubonos, I. V. Grigorieva, and A. A. Firsov, Science 306, 666 (2004).

${ }^{2}$ K. S. Novoselov, D. Jiang, F. Shedin, T. J. Booth, V. V. Khotkevich, S. V. Morozov, and A. K. Geim, Proc. Natl. Acad. Sci. U.S.A. 102, 10451 (2005).

${ }^{3}$ A. K. Geim and K. S. Novoselov, Nature Mater. 6, 183 (2007).

${ }^{4}$ N. Tombros, C. Jozsa, M. Popinciuc, H. T. Jonkman, and B. J. van Wees, Nature (London) 448, 571 (2007).

${ }^{5}$ M. Y. Han, B. Özyilmaz, Y. Zhang, and P. Kim, Phys. Rev. Lett. 98, 206805 (2007).

${ }^{6}$ T. Ohta, A. Bostwick, T. Seyller, K. Horn, and E. Rotenberg, Science 313, 951 (2006).

${ }^{7}$ E. McCann, Phys. Rev. B 74, 161403 (2006).

${ }^{8}$ D. C. Elias, R. R. Nair, T. M. G. Mohiuddin, S. V. Morozov, P. Blake, M. P. Halsall, A. C. Ferrari, D. W. Boukhvalov, M. I. Katsnelson, A. K. Geim, and K. S. Novoselov, Science 323, 610 (2009).

${ }^{9}$ S. H. Cheng, K. Zou, F. Okino, H. R. Gutierrez, A. Gupta, N. Shen, P. C. Eklund, J. O. Sofo, and J. Zhu, Phys. Rev. B 81, 205435 (2010).

${ }^{10}$ F. Withers, M. Dubois, and A. K. Savchenko, Phys. Rev. B 82, 073403 (2010).

${ }^{11}$ R. Nair, W. Ren, R. Jalil, I. Riaz, V. Kravets, L. Britnell, P. Blake, F. Schedin, A. Mayorov, S. Yuan, M. Katsnelson, H. Cheng, W. Strupinski, L. Bulusheva, A. Okotrub, I. Grigorieva, A. Grigorenko, K. Novoselov, and A. K. Geim, arXiv:1006.3016, Small (to be published).

${ }^{12}$ D. A. Dikin, S. Stankovich, E. J. Zimney, R. D. Piner, G. H. B. Dommett, G. Evmenenko, S. T. Nguyen, and R. S. Ruoff, Nature (London) 448, 457 (2007).

${ }^{13}$ G. Eda and M. Chhowalla, Adv. Mater. 22, 2392 (2010).

${ }^{14}$ M. H. F. Sluiter and Y. Kawazoe, Phys. Rev. B 68, 085410 (2003).

15 J. O. Sofo, A. S. Chaudhari, and G. D. Barber, Phys. Rev. B 75, 153401 (2007).

${ }^{16}$ J. T. Robinson, J. S. Burgess, C. E. Junkermeier, S. C. Badescu, T. L. Reinecke, F. K. Perkins, M. K. Zalalutdniov, J. W. Baldwin, J. C. Culbertson, P. E. Sheehan, and E. S. Snow, Nano Lett. 10, 3001 (2010).

${ }^{17}$ Graphite fluorides were first synthesized in 1934 [O. Ruff and O. Bretschneider, Z. Anorg. Allg. Chem. 217, 1 (1934)]. However, only recently (see Refs. 10 and 16) it was possible to isolate monolayers of this material. This implies a transition from a three-dimensional to a two-dimensional material similar to the transition from graphite to graphene.
${ }^{18}$ J.-C. Charlier, X. Gonze, and J.-P. Michenaud, Phys. Rev. B 47, 16162 (1993).

${ }^{19}$ M. Klintenberg, S. Lebègue, M. I. Katsnelson, and O. Eriksson, Phys. Rev. B 81, 085433 (2010).

${ }^{20}$ E. Muñoz, A. K. Singh, M. A. Ribas, E. S. Penev, and B. I. Yakobson, Diamond Relat. Mater. 19, 368 (2010).

${ }^{21}$ C. Lee, X. Wei, J. W. Kysar, and J. Hone, Science 321, 385 (2008).

${ }^{22}$ F. Liu, P. Ming, and J. Li, Phys. Rev. B 76, 064120 (2007).

${ }^{23}$ J. P. Perdew, K. Burke, and M. Ernzerhof, Phys. Rev. Lett. 77, 3865 (1996).

${ }^{24}$ H. J. Monkhorst and J. D. Pack, Phys. Rev. B 13, 5188 (1976).

${ }^{25}$ N. Troullier and J. L. Martins, Phys. Rev. B 43, 1993 (1991).

${ }^{26}$ X. Gonze, J.-M. Beuken, R. Caracas, F. Detraux, M. Fuchs, G.-M. Rignanese, L. Sindic, M. Verstraete, G. Zerah, F. Jollet, M. Torrent, A. Roy, M. Mikami, P. Ghosez, J.-Y. Raty, and D. C. Allan, Comput. Mater. Sci. 25, 478 (2002).

${ }^{27}$ A. Marini, C. Hogan, M. Grüning, and D. Varsano, Comput. Phys. Commun. 180, 1392 (2009).

${ }^{28}$ L. Hedin, Phys. Rev. 139, A796 (1965).

${ }^{29}$ C. A. Rozzi, D. Varsano, A. Marini, E. K. U. Gross, and A. Rubio, Phys. Rev. B 73, 205119 (2006).

${ }^{30}$ S. S. Han, T. H. Yu, B. V. Merinov, A. C. T. van Duin, R. Yazami, and W. A. Goddard, Chem. Mater. 22, 2142 (2010).

${ }^{31}$ O. Leenaerts, B. Partoens, and F. M. Peeters, Phys. Rev. B 80, 245422 (2009).

${ }^{32}$ M. Z. S. Flores, P. A. S. Autreto, S. B. Legoas, and D. S. Galvao, Nanotechnology 20, 465704 (2009).

${ }^{33}$ O. Leenaerts, B. Partoens, and F. M. Peeters, Appl. Phys. Lett. 92, 243125 (2008).

${ }^{34}$ P. Bulltinck, C. Van Alsenoy, P. W. Ayers, and R. Carbo-Dorca, J. Chem. Phys. 126, 144111 (2007).

${ }^{35}$ F. L. Hirshfeld, Theor. Chim. Acta 44, 129 (1977).

${ }^{36}$ The Young's modulus and Poisson's ratio are determined by stretching one of the supercell parameters $\left(a_{x}, a_{y}\right)$ by $0.5 \%$ and subsequent relaxation of the other supercell parameter and all the atomic posisitions.

${ }^{37}$ M. Topsakal, S. Cahangirov, and S. Ciraci, Appl. Phys. Lett. 96, 091912 (2010).

${ }^{38}$ H. Şahin, C. Ataca, and S. Ciraci, Phys. Rev. B 81, 205417 (2010).

${ }^{39}$ M. F. Thorpe and P. N. Sen, J. Acoust. Soc. Am. 77, 1674 (1985).

${ }^{40}$ N. Lu, Z. Li, and J. Yang, J. Phys. Chem. C 113, 16741 (2009).

${ }^{41}$ J. Berashevich and T. Chakraborty, Nanotechnology 21, 355201 (2010).

${ }^{42}$ Y.-J. Yu, Y. Zhao, S. Ryu, L. E. Brus, K. S. Kim, and P. Kim, Nano Lett. 9, 3430 (2009). 